\title{
The Metaphysics of Possible Worlds in the Narrative of Fatos Kongoli Time and Symmetric Space in the Comparison between Kongoli - Buzzati
}

Eris Rusi

\author{
"Fan S. Noli" University of Korça, Albania \\ Faculty of Education and Philology \\ email: erusis1@yahoo.com
}

\section{Doi:10.5901/mjss.2013.v4n9p512}

\section{Abstract}

The following paper aims to take a virtual trip to the author's literary work, Fatos Kongoli, and treating some of the most important themes of his narrative. The author, selected for this paper, is a contemporary Albanian writer who manages to fill with anxiety and spirituality the words, the narrative voices and the variations of scenarios.By analyzing some of the most important aspects of the work of Kongoli, research will focus on literary and human development of this author, on the limits of his research that lies beyond the ideas and reach the major topics like Life, Time and Death, wrapped in the individual level as well as the general one of all humanity. This paper among others will propose a comparison between Fatos Kongoli and Dino Buzzati, aiming a symmetric approach between these two authors. The research, in this sense, will be accomplished by following some reading keys that refer to:

- Penetration of human life;

- The real world and the world of the possible, as symmetric representations of life and death;

- Moral and immoral earthly issues;

- Transiency and eternity;

- Illustration of fantastic dimension and the dimension of reality;

- De-codification of earthly and divine meaning in affective relationships.

The comparison between Kongoli and Buzzati will be conducted from the perspective of the analysis of these two authors, as people destined to be pushed into the distant lands of the imagination. The essential would be to be recognized the filters on topics of reflection of human enigma, to penetrate in the metaphysical battle against Being as Time-consuming.

Keywords: metaphysics, literary contemporary, symmetrical comparison, symmetrical Time, fantastic dimension, transiency, eternity.

\section{Introduzione}

\author{
Ognuno sta solo sul cuor della terra \\ trafitto da un raggio di sole: \\ ed è subito sera! \\ Salvatore Quasimodo
}

La letteratura albanese è come una piccola barca, con dentro pochi coraggiosi rematori che ordinano di salire su, e poi traghettano i passeggeri in un fiume misterioso, dove tutto ricorda la Vita e le sue ombre notturne. Ci si riesce a sentire il battito del cuore, i pensieri che echeggiano nel silenzio, i sogni che si arrampicano fino all'orlo della realtà.

I personaggi che si incontrano sembrano cosi reali da non osare a toccarli con la mano, e nello stesso tempo si ha la sensazione di una realtà effimera, dove tutto può scomparire da un momento ad altro.

Dentro quest'ambiente vive e respira professionalmente Fatos Kongoli, l'autore prescelto per questa ricerca, uno scrittore che dopo gli anni '90 si presentò con un impatto importante nella letteratura albanese, ornando con una spiritualità quasi perduta dopo il comunismo, le parole dei suoi scritti.

Attraverso la sua opera si può perlustrare lo sviluppo letterario e umano dell'autore, i confini della sua ricerca, la triplice matrice della sua volontà artistica e il suo sfogo a dipingere con le proprie idee, la Vita, il Tempo e la Morte di se stesso e dell'intera umanità.

Vorrei mettere in chiaro il mio metodo di interpretare Kongoli, citando un momento della biografia del pittore Paul Klee. Nel suo diario Klee aveva scritto che all'età di nove anni andava nella trattoria dello zio, dove:

“...c'erano tavoli con piani di marmo levigato, la cui superficie era, per vetustà, un intrico di solchi. In questo labirinto di 
linee si potevano vedere grottesche figure umane e fissarle con la matita" ( Paul Klee, 2010).

Questo vedere nei solchi della superficie del marmo figure diverse è, per dirla con Wittgenstein delle Osservazioni sulla filosofia della psicologia, un vero e proprio "vedere come":

"...un intrico di linee improvvisamente si anima e in esse scorgiamo una figura, un volto, una fisionomia. L'immagine che ci appare è il risultato non della visione retinica, ma di un "vedere come", di uno "sguardo", con il quale di "colpo" cogliamo qualcosa che rimaneva nascosto alla vista e che non possiamo indicare senza riferirci proprio a quell'intrico di linee. Questo vuol dire che quelle linee possono dare luogo a una molteplicità di rappresentazioni nessuna delle quali è prevedibile..."(Giuseppe di Giacomo, 2005).

Sotto quest'ottica di valutazione, i personaggi tratti dai libri di Kongoli mostrano diverse sfacciature dell'intimo umano, si lasciano cadere per rialzarsi in realtà diverse, dove i confini fra il mondo dei sogni e la quotidianità terrestre si sfumano tra loro.

Come si presenta Kongoli dentro la cornice biografica della sua vita?

Nato nel 1944 a Elbasan, nell'Albania Centrale, ha studiato matematica a Pechino e poi a Tirana, dove si è laureato nel 1967. A prima vista nascono i dubbi da questa convivenza particolare tra la matematica e la letteratura nella sua formazione, ma lui stesso chiarisce ogni titubanza sostenendo che:

\begin{abstract}
"Il legame si crea spontaneamente, come nel mio caso in cui dopo aver avuto una formazione matematica di base, è stata un'inclinazione del tutto intima a spingermi verso la letteratura. La matematica se non altro dà all'uomo ciò che comunemente è detta 'logica matematica'. Questo genere di logica non ti permette "di volare". Ė questa logica che interviene spontaneamente e si riflette nella struttura dell'opera sino alla costruzione della frase stessa e via di seguito. D'altra parte, esiste un certo legame a livello universale tra letteratura, arte e scienze: Einstein ha detto che, nello scoprire la teoria della relatività, si è ispirato, tra gli altri, a Dostoevskij"(Rivista Amaltea, 2008).
\end{abstract}

Kongoli ha lavorato a lungo come giornalista e capo redattore presso la casa editrice "Naim Frasheri" di Tirana. Sotto la dittatura comunista ha pubblicato solo due romanzi Noi tre (1985), Il carosello (1990) e subito dopo la caduta del comunismo espresse la sua intenzione di scrivere una tetralogia. Si rammenta in particolare un ciclo di cinque romanzi, Un uomo dall'nulla (1992), L'ombra dell'altro (1994), Il drago d'avorio (1999), Il sogno di Damocle (2000), Pelle di cane (2003), chiamati dall'autore "Le carceri della memoria" e dedicati all'ultima fase della dittatura in Albania, alla sua caduta ed ai problemi esistenziali che ne conseguirono nella fase di transizione postcomunista.

Queste opere sono il tentativo di oltrepassare l'osservazione della quotidianità grigia albanese, cercando il dialogo tra il passato e il presente nella concezione dell'autore. Attraverso "Le carceri della memoria" Kongoli sconfina nel campo della riflessione sui temi e problemi fondamentali dell'uomo in rapporto alla sua condizione esistenziale, ai suoi simili, alla morte e a Dio. La scrittura particolare che s'intravede in questi libri, difatti favorisce l'esplicarsi di un'immaginazione che, negli scritti di Kongoli sembra di sondare quanto di misterioso e oscuro caratterizza il reale.

Ciò che si crea nell'immaginario del lettore è la sensazione di un silenzio ingombrante, onnipresente; le pause, dove sembra che non succeda niente e tutto accade senza accorgersene, sembrano degli elementi quasi irreali, fantastici, con i quali uno comincia ad abituarsi.

Sotto questa luce, si possono vedere delle interessanti affinità tra Kongoli e Dino Buzzati. Anche quest'ultimo ebbe la necessità di guardare il Silenzio come un Deserto, dove tutto si spegneva sotto i fuochi del sole e della vita che colpivano e costringevano le sagome umane ad aspettare che tutto cambiasse attraverso i miracoli.

L'anormalità e l'angoscia sono elementi della narrativa che avvicina Buzzati a Kongoli. II lettore esce da alcuni romanzi dello scrittore albanese come da un risveglio. Ora sa che prima sognava. Questo tipo di scrittura assomiglia all'esplorazione del tempo. II viaggio nel passato e nella memoria è un punto fermo dove la realtà si converge nell'illusorio esistenziale.

Kongoli allora è un esploratore contemporaneo del suo tempo?

C'è da ricordare che dopo la caduta del comunismo in Albania, erano in molti gli autori che decisero di dare un taglio netto al passato e ripartire. Tra questi, Kongoli scelse il momento felice di partire, per non tornare più. Attraverso il racconto del passato, lui mette in luce una vita che nasce e poi si disfa di tutto ciò che ha intorno a se.

Chiamando in causa Dino Buzzati, il percorso metafisico di Kongoli assomiglia al cammino impreciso del messaggero:

"...Sia come sia o messaggero, porta la notizia che io vengo, non occorre neanche che tu ti faccia vedere ancora. 
Questa sera mi sento veramente bene, sebbene i pensieri ondeggino un poco, e ho preso la decisione di partire (Ma sarò poi capace? Non farà storie poi la mia anima, al momento buono non si metterà a tremare, non nasconderà la testa tra le pavide ali, dicendo di non andare più avanti?"(Dino Buzzati, 1999).

Un viaggio, un'esplorazione, che porta i lettori verso un pellegrinaggio mistico, letterario, umano, nelle pagine scritte di Kongoli, dove si prova una sensazione strana, misteriosa, che riempie l'anima e incoraggia ad andare avanti, a vedere oltre, per cercar di capire, se è possibile, qualcosa di più della quotidiana realtà.

\section{Dai viaggi esistenziali alla parabola della morte. I segni della lotta quotidiana contro l'ombra di noi stessi}

I ricordi che ritornano, prima frequentemente, poi sempre più di rado, e quindi a intervalli di anni e di decenni, sono i legami che ci uniscono al tempo mitico della nostra fanciullezza, della nostra infanzia, o comunque, che ci uniscono alla nostra vita passata. Legami di un mondo raccolto e fatato, che si allentano inesorabilmente di anno in anno, fino al punto che non possono essere più nemmeno interpretati, quando eccezionalmente arrivano alla nostra riflessione. L'aria mistica che si respira sorvegliando il tempo, rimanda spesso a un'esistenza felice, ti fa pensare a persone che hanno gioito aggrappati all'illusione delle loro vite.

Quest'atmosfera si capovolge nel romanzo "L'ombra dell'altro" di Kongoli. II Protagonista, Festim Gurabardhi, vive un'esistenza tormentata, costretto a conoscere da bambino il perseguitore che segnerà ogni momento cruciale della sua vita.

La storia è ambientata durante il regime comunista in Albania, ma il protagonista è destinato a vivere tra le spoglie di un'infelicità persistente. Festim Gurabardhi ha una vita svuotata da ogni illusione temporanea della gioia, lavora in una casa editrice, dove filtra i manoscritti in base ai parametri voluti dal regime, si tufa in relazioni temporanee con donne che scivolano come sagome trasparenti dalla sua vita e nel silenzio che si apre davanti ai suoi pensieri, cerca di arrivare senza convinzione al domani.

Quali sono i suoi ricordi da bambino? La morte dei genitori, il quasi annegamento all'età di dodici anni, il fratello dal nome Abele, che dopo essersi suicidato gli farà pensare sempre di più al Caino biblico che sente di rappresentare.

Non ci sono descrizioni minuziose di cose belle e leggere dell'infanzia. La nonna che si prende cura di lui è solo un elemento di racconto come tanti altri, anche lei muore, e tutti i famigliari che Festim impara a conoscere, muoiono, fanno una brutta fine o semplicemente svaniscono in silenzio.

C'è da dire che questi ricordi che assomigliano al tentativo di evocare l'inferno fanciullesco dentro la realtà infernale, sono come dei testimoni molesti e ingombranti. Festim Gurabardhi non prova quasi mai "rimpianto delle gioie lasciate", ma attende senza poter far niente, e lascia scorrere via, nel fiume del tempo, la propria esistenza.

Lui conosce la persecuzione dai primi anni di vita sotto il nome di Valmir, che nel trascorrere degli anni sarà per sempre un'ombra ingombrante dietro le pieghe esistenziali di Festim. L'Albania del regime comunista era piena di gente come Valmir, che si mettevano al servizio della macchina statale per mietere paura e dolore nelle anime tormentate delle persone.

Valmir è il male, e ogniqualvolta che s'incrocia con Festim, l'aria diventa pesante e la morte si avvicina curiosa a loro. Valmir è quello che gli fa vedere per la prima volta la faccia della morte a Festim, quando lui era ancora un ragazzino:

"Valmir mi mostrò la faccia della morte. Questo coincide con l'epoca in cui c'erano le impiccagioni pubbliche in piazza e gli impiccati erano lasciati fluttuare in aria come pendoli, per il tempo necessario che serviva a tutta la gente di vederli"(Kongoli, 1999).

Il bambino Valmir non si accontenta di ciò che vede. Lui osa e vuole provare cosa significa maneggiare la morte. Così inscena il genocidio dei gatti del quartiere:

"Il modo in cui Valmir uccise il gatto nero del sotterraneo mi sconvolse profondamente. Prima gli staccò la testa con un coltello. Poi gli aprì la pancia e cosi fece uscire l'intestino. Le sue mani si sporcarono di sangue e dalla pancia del gatto si sprigionò un'aria cattiva.[...] Quella notte gridai nel sonno. Mi sembrava come se un essere senza volto m'inseguisse con un coltello in mano. lo mi allontanavo, ma ero convinto che se mi avesse preso, mi avrebbe tagliato la testa, mi avrebbe aperto la pancia per tirare fuori il mio intestino".

Ci troviamo davanti a una realtà che assomiglia all'incubo e incubi presenti quanto la realtà. II bambino Valmir mistifica il male, si sottopone al regime comunista e fa la spia per arrivare alle condanne della gente comune. II 
passaggio dal mondo dei bambini a quello dei grandi si fa indolore per lui, che si trasforma nell'inquirente Valmir D., alienato dal regime totalitario e portatore di una Maledizione continua per i comuni mortali.

L'inquirente del regime sceglie la sua vittima, la pedina, la tortura dal punto di vista psicologico e quanto entra nei suoi sogni intimi, le uccide le speranze soffocando ogni luce dei pensieri.

Valmir D. Ė un cacciatore di anime perdute, sempre alla ricerca della rovina casuale di singoli sconosciuti.

Ritornando al paragone con Dino Buzzati, si trova un'analogia con il racconto "La bomba atomica", dove il male sceglie in modo indistinto le sue vittime.

In questo racconto è la Morte che si diverte a fare visite a domicilio, e non solo le voci preoccupate degli inquilini, ma anche i sogni premonitori e le telefonate degli sconosciuti, fanno alzare dal letto il Prescelto, il Predestinato, che si vergogna di uscire in pigiama a vedere che succede, ma poi, alla fine, spinto da un misterioso desiderio di scoprire di che si tratta, fa il passo decisivo in avanti.

La morte è il momento della verità, e quelli che sentono di averla fatta franca, tolgono via le maschere dalle proprie facce e si danno ai festeggiamenti; brindano alla malasorte capitata ad un altro. Insomma, piccoli esseri umani, meschini, senza la minima voglia di esibire un sentimento di pietà, per qualcuno che sta per "andare via".

È il mondo di tutti i giorni, sono le persone che ci salutano la mattina al bar, ma quando si tratta di togliere le maschere, si vede una nudità mostruosa di anime che si strusciano, dietro le pareti, per non farsi prendere, per non farsi vedere.

Il campanello della Notte ha un suono particolare, differente, da persona a persona. Solo dinanzi a questo bivio, si riesce a capire la solitudine della vita umana, un correre senza fiato in un deserto d'illusioni, la scalata nella cima più alta del mondo per morire poco prima di esserci riusciti.

\footnotetext{
"...La gente mi fissava. Mai vidi volti umani stravolti da una felicità così selvaggia. Uno non seppe resistere e scoppiò in una risata che fini in una tosse cavernosa: era il vecchio Percalli, quello dei tappeti all'asta. Capii. Il cassone con l'inferno dentro era per me, un esclusivo dono, per me solo. E gli altri erano salvi"(Giulio Carnazzi, 1998).
}

Si tratta di singole esistenze umane, delle quali si sa poco o niente. Buzzati impone il paragone fra una vita pensata, dove ognuno è padrone della situazione e non si lascia mai andare, e la realtà così com'è, dove il Tempo, un vento che soffia sopra le vittime prescelte, non lascia nessun briciolo di speranza.

Kongoli nel suo libro offre la stessa idea del male che si incarna nel inquirente Valmir D. Questo è l'ombra degli altri, si mette nella scia delle vite anonime con l'intento di distruggerle e ci riesce malvagiamente nella sua intenzione.

Le ambientazioni del libro assomigliano a un paradosso tra il sogno e la realtà, con l'eccezione del protagonista, tutti gli altri personaggi sono sfumati nei loro piccoli sforzi di sopravvivere. Klea, Gusti, Sara, Hektor, come tanti altri hanno il destino segnato dalla fatalità che arriva e non gli da scampo. Non possiamo sapere molto di loro, si capisce, non dobbiamo affezionarci alla loro avventura. Tanto è tutto perduto.

Tutti hanno in comune una sola data, quella della loro morte, e davanti a un evento così straziante, non ci resta che pensare e valutare le piccole inutili cose che facciamo periodicamente. È come se Kongoli ravvisasse nella vita di tutti giorni, la fine comune che ci aspetta, senza aver la possibilità di scelta. Un'interpretazione squallida, si può dire, e meschina, della sorte degli uomini sulla piccola terra albanese e comunista.

Lo stesso Festim Gurabardhi si trascina nel proprio destino, si trasforma in un cadavere vivente, con la paura di incrociare il proprio esecutore in ogni passo che incammina per la strada.

La morte in sé, diventa una cosa assurda, non ha più senso parlare di destini già segnati. Un morto vivente diventa un estraneo, non trova più pace se cerca di rientrare nella vita di tutti i giorni. Con un po' di amarezza Kongoli ci fa sentire sempre partecipi di movimenti universali, dove lottando a fianco degli sconosciuti, si combatte, infine, forse inutilmente e senza troppe pretese, anche per noi stessi.

\section{II mondo reale e il mondo possibile, come rappresentazioni simmetriche della vita e della morte}

II titolo metaforico del libro "pelle di cane" di Kongoli, offre la possibilità di entrare nelle varie sfumature identitarie, psicologiche e spirituali del protagonista Kristi Tarapi. Questo è un personaggio che s'identifica contemporaneamente come una persona normale e straordinaria, le sue allucinazioni offrono una visione chiara e distinta della vita, i suoi passi sprofondano tra la sabbia terrestre e le nuvole del cielo.

Ciò che impressiona la mente del lettore è l'idea di trovarsi di fronte a un uomo misero, solitario, triste, egoista, quasi antisociale, disperato, disprezzato, ma comunque pieno di sentimenti quasi irreali che si schiariscono quando la vita si spezza tra il sogno e la realtà. 
Durante tutto il racconto, il protagonista crede di sentire il suono di una marcia funebre che lo marca strettamente nei suoi spostamenti. Si tratta della processione organizzata per il suo funerale, e tra gli altri elementi che rafforzano questa visione, spicca la presenza di Hades, la divinità greca dei morti, il Dio Plutone dei Romani per intenderci, che prende il volto indistinto di una presenza che sembra esistere nel mondo dei vivi dall'inizio dei secoli.

I personaggi di questo libro evocano al lettore i vizi e i desideri che ognuno porta dentro di sé, nel sotterraneo dell'anima, senza la forza o la possibilità di mostrarle al mondo di fuori che guarda scrupolosamente la vita. Questo romanzo assomiglia a un viaggio nel tempo, dove ci si sposta avanti e indietro, nell'infanzia del protagonista e nelle proiezioni buie dell'uomo maturo che sente e immagina da vivo il suo funerale.

Il protagonista Tarapi racconta sempre in prima persona, e riesce a dare l'atmosfera dell'Albania sotto il regime comunista, dove le persecuzioni e il dolore provato costantemente fano evocare le catacombe dei morti antichi nel regno di Hades.

Questa sensazione si fa viva anche dopo, quando lui cresce e lo specchio gli fa vedere ogni giorno la faccia stanca di un uomo sofferente. Anche allora le storie vissute continuano a rimanere vive e a sorgere violentemente nei sogni e nei ricordi. Di quanto in quanto emergono anche le immagini dei primi amori, il mondo femminile entra e prende il palcoscenico dei ricordi. Le donne rappresentano il volto bello del mondo, cosi è per Liza, per Dolores, per Lori, e ciò che si comprende è che il protagonista in tutta la sua vita è riuscito ad amare unicamente una dona sola.

Infatti Dolores è la figlia di Liza, e Lori è la figlia di Dolores: sembra di assistere a una ripetizione del mondo, a un procedimento ciclico, dove sempre e comunque Kristi Tarapi si anima di sentimenti e prova gioia e disperazione in ogni singola relazione successiva nel tempo.

Lui, comunque, rimane un protagonista molto complesso, si autodefinisce "pelle di cane", come se questo pseudonimo fosse abbastanza esauriente da rappresentare un uomo senza ideali, senza alcun vincolo morale e fede nei altri, spogliato dalle preoccupazioni pubbliche e sociali, e solamente interessato alla sua crisi esistenziale.

Lui è un padre che non sa fare il padre, che sogna di fare l'amore con l'amica della sua figlia mentre sua moglie tossisce gli ultimi istanti della vita, è un marito indegno che si nutre del vuoto che accompagna le sue giornate.

Abbiamo difronte un protagonista che si fa fatica a comprendere, nella vita di tutti i giorni sarebbe qualcuno cui non gli spetterebbe il minimo saluto di cortesia, ma il mondo raccontato da Kongoli in questo libro è pieno di paradossi, e per di più assomiglia all'inferno (Hades che si vede dall'inizio alla fine del libro attesta molto questa percezione).

L'autore offre una riflessione interessante sull'angoscia e sulla perdita di armonia con noi stessi e con il mondo attorno, raccontando la vita di un personaggio smarrito, che vive tra il passato e il presente, con i ricordi degli anni lasciati alle spalle che minacciano la sua abitudine di esistere senza chiedere nulla al mondo.

La vita misera e meschina del protagonista, piena di sofferenze e sotto la stretta morsa del regime comunista è la rappresentazione del mondo reale dove lui si muove. Di tutt'altra intensità invece, si presenta la vita immersa nei sogni e nei ricordi, dove anche le situazioni più fantasmagoriche sono narrate con una tranquillità convincente come se si trattasse di cose che si possono vedere e toccare con la mano tutti i giorni. Quest'altra dimensione della vita assomiglia al mondo possibile, dove il protagonista si avvicina al latto meno buio della vita.

In questa direzione la narrazione si smuove dalla realtà, verso un approfondimento fantastico della storia. Ovviamente quest'approccio fantastico non si colloca al di fuori della realtà, ma si ancora saldamente a fatti veri, si insinua in zone conosciute e quotidiane fornendo della realtà immagini deformate e straordinarie, portando così l'ignoto nel noto e nel familiare.

II fantastico Di Kongoli presenta un aldilà interiore, dove Hades-Plutone rispecchia l'ombra della solitudine e l'ansia del protagonista in balia delle forze estranee che lo sottomettono.

"Hades stava in piedi sopra le scale di marmo, in una posa statuaria, con una mano appoggiata vicino al piedistallo vuoto. La sua testa si mosse improvvisamente. Hades, statuario e cerimoniale, aveva preso quella posizione come se volesse rialzarsi sul piedestallo, con una mano che indicava un punto indistinto nello spazio.

In realtà la sua mano segnava una direzione molto chiara, verso est, dove si trovava il cimitero, ma questo l'ho capito più tardi" (Kongoli, 2008).

Questo tentativo estremo di spingere l'angoscia verso il limite della follia, con la presenza di Hades prima e dopo, fa del protagonista stesso l'oggetto inquietante, che si offre al lettore come un uomo con i suoi sogni, le sue allucinazioni, le sue pulsioni inconsce, le sue incertezze, i suoi smarrimenti.

Si possono notare elementi di realismo magico nella lettera del libro. Riferendosi a Luis Leal, la trasformazione del comune di ogni giorno in qualcosa di fantastico e ireale è: 
"un atteggiamento verso la realtà che si può esprimere in modi popolari o sofisticati, in stili elaborati o rustici, in strutture chiuse o aperte. [...] l'autore confronta la realtà e cerca di liberarla, di scoprire cosa c'è di misterioso nelle cose, nella vita, nei comportamenti umani. [...]

Il realismo magico non cerca di copiare la realtà circostante...o di sconvolgerla...ma solo di misurare il mistero che respira dietro le cose"(Luis Leal, 1995).

Attraverso il suo racconto Kongoli crea un mondo che, dopo, esiste ancora e non può più essere trascurato. Kongoli richiama nel mondo possibile della sua esistenza, Hades, II Dio Archetipe della Morte. Anche per Giorgio Barberi Squarotti, il fantastico è "Io spazio in cui più pienamente si esercita il potere mitopoietico della letteratura: inventando, appunto, altri e diversi mondi, che inevitabilmente, dopo, esistono, ma anche recuperando la serietà di fondo di quel gioco che è la letteratura" (G.B.Squarotti, 1981).

In fatti c'è quasi la sensazione di un gioco continuo tra Hades e il protagonista. Non si sa bene per chi si organizza di volta in volta il funerale. Le voci che Hades è morto vengono soverchiate dalla notizia della morte del protagonista, ma quando i due si incrociano, oltre alla smorfia, si crea la sensazione di uno scherzo di cattivo gusto, che può continuare per l'eternità.

Lo smalto (o il segreto) della narrazione in questo libro, sta nella quasi -banalizzazione del fantastico, nel rovesciare cioè il guanto della cronaca, sospingendo lievemente, col minimo artificio stilistico e concettuale, il dato reale sul precipizio dell'ireale, il momento generico sull'interrogazione generica. Kongoli si avvicina in questa direzione ancora una volta a Dino Buzzati.

La sua dimensione fantastica, Buzzati stesso lo ripeteva nell'intervista concessa a Yves Panafieu, enunciando le regole cui si era tenuto nella stesura delle sue novelle:

"Io, raccontando una cosa di carattere fantastico, devo cercare al massimo di renderla plausibile ed evidente. E quando dico io, in realtà implico qualsiasi altro scrittore di tipo fantastico. Per questo, secondo me, la cosa fantastica deve essere resa più vicina che sia possibile, proprio, alla cronaca. La parola giusta non è "banalizzare", ma insomma è un po' questo. Voglio dire che, affinché una storia fantastica sia efficace, bisogna che sia raccontata nei termini più semplici e pratici. Anzi, quasi burocratici. (Y.Panafieu, 1973).

\section{L'uomo, l'artista e lo spettatore}

T.S. Eliot, nel suo saggio Tradition and Individual Talent, scrive:

"L'artista sarà tanto più perfetto quanto sarà completamente separato in lui l'uomo che soffre e lo spirito che crea; e tanto più perfettamente lo spirito digerirà e trasmuterà le passioni che sono il suo elemento"(Eliot, 2010).

Fatos Kongoli nel 2010 pubblicò un libro che aveva il compito di liberarlo dal turbamento nascosto dentro l'anima. Si tratta del racconto autobiografico "Illusioni nel cassetto" che lo stesso autore lo definisce come un "quasi romanzo" che mette in luce la voglia di confessare un atto del passato, quando l'autore, messe alle strette dal regime comunista, fu obbligato a deporre in tribunale contro un altro scrittore che fu condannato da innocente.

Oltre questo fatto, ciò che incuriosisce nel libro è la forma plastica, in movimento, della memoria. Un biografo pretenderebbe di conservare la verità di ciò che è successo, mentre Kongoli interviene e con la libertà dello scrittore, trasforma in personaggi letterari molti dei protagonisti reali della sua vita.

La memoria di Kongoli funziona quando da una forma ben definita ai fatti e alla materia dei ricordi, selezionando il passato per assicurare così alla sua vita un ordine che nella realtà è difficile da trovare.

Kongoli insiste quando dice che è nato senza talento. La musica non era la sua predilezione, e tra i suoi compagni d'infanzia era uno qualsiasi fra i tanti. Sopranominato "dentone" per la fisionomia della sua bocca, abbastanza da sentirsi disgraziato, Kongoli rappresenta le qualità del protagonista senza voler essere tale.

I nomi e i personaggi citati riaffiorano volta per volta come citazioni reali, per convincere il lettore sulla verità dei fatti raccontati. L'ambientazione dominante riguarda l'Albania comunista, gli anni vissuti sotto il regime, accompagnati da ordini e sentimenti collettivi, dalle direttive e dall'obbedienza cieca di seguire in fondo la fatalità dell'esistenza.

Ancora prima di essere pubblicato, il libro suscitò molte polemiche nella stampa albanese. In molti alzarono la voce chiedendo di definire la posizione di Kongoli come scrittore durante il regime dittatoriale, il suo ruolo e la responsabilità che avrebbe avuto con le sue testimonianze.

Ma è lo stesso autore che scende se stesso dal piedestallo del tempo. Lui racconta, e fa entrare la luce nei fatti buoi del passato. La sua confessione è una liberazione, per un fatto, dove dagli archivi del tribunale si evidenzia che la 
sua testimonianza era irrilevante ai fini del processo.

Si evince dalle pagine del libro la voglia di liberarsi da quelle piccole e fastidiose preoccupazioni che noi rimandiamo dentro l'anima. Tornando al paragone Kongoli -Buzzati, anche quest'ultimo ebbe la voglia di confessare la sua inquietudine legata alla continua comparazione tra lui e Franz Kafka. Si può intuire una sofferenza psicologica di Buzzati, una lotta progressiva dentro di lui, fra l'uomo con le proprie smanie e l'artista, specialmente quando questi paragoni sono insistenti, quasi ossessivi nella lettura delle sue opere. Le ripetute volte che la critica gli attribuisce legami profondi e temi uguali con lo scrittore boemo, danno profondamente fastidio a Buzzati.

Ecco allora la sua confessione:

"Da quando ho cominciato a scrivere Kafka è stato la mia croce. Non c'è stato mio racconto, romanzo, commedia dove qualcuno non ravvisasse somiglianze, derivazioni, imitazioni o addirittura sfrontati plagi a spese dello scrittore boemo. Alcuni critici denunciavano colpevoli analogie con Kafka anche quando spedivo un telegramma o compilavo il modulo Vanoni.

Tutto questo da molti anni ha determinato in me, nei riguardi di Franz Kafka, non un complesso di inferiorità, ma un complesso di fastidio." (Carnazzi, 1998).

E poi testimonia che da allora, non ha voluto più leggere cose sue, né biografie, né saggi che lo riguardavano. Ma quando si trova e Praga fa il percorso dove ha vissuto Kafka, alla fine si ferma alla tomba di quest'ultimo. Qui l'orgoglio si placa, lo scrittore si mette in pace con se stesso, guarda i sassi a due, sul bordo del sepolcro, l'omaggio degli ebrei ai loro morti. II ricordo dell'antico deserto.

Le confessioni di Kongoli e Buzzati mostrano la timidezza, l'insicurezza che a volte hanno le persone generose. $\mathrm{E}$ sono anche queste, certe forme quasi nascoste delle loro personalità, che rendono i due amati, voluti dai lettori e capaci di dare speranza e sorriso, meditazione e immaginazione, attraverso la loro vita e i loro scritti.

\section{Conclusione}

L'impulso vitale di Kongoli è "fatto di quell'impalpabile sostanza che volgarmente si chiama favola o illusione: anche se è vero". Lui ha sempre scritto la stessa cosa; la sua vita, anzi, le sue vite come un "romanzo a puntate" e le sue esperienze quotidiane e interiori, veglianti e oniriche.

Facendo una copertura di alcune opere di Kongoli, ho tentato di dare informazioni anche sulla vita che si faceva e l'aria che circolava nell'Albania comunista durante 50 anni di regime, dove spesso e volentieri mancava il coraggio di raccontare direttamente le cose, così che i libri dove la storia sembrava assente, erano numerosi e spolveravano le vetrine delle biblioteche di tutto il paese. Romanzi scritti sul passato e sul presente, si mostrano nella loro magica essenza cifrata, si trasformano in riflessioni sulla storia e sulla parola, sul destino dell'uomo su questa terra e oltre la nera porta della Morte. II puro, il vero, l'immutabile è possibile solo attraverso l'esperimento magico dell'arte.

\section{References}

Paul Klee, Diari, Il saggiatore, Milano 2010

Giuseppe Di Giacomo, Introduzione a Klee, Laterza, 2005

Dino Buzzati, Sessanta racconti, in Opere scelte, a cura di Giulio Carnazzi, Milano 1998

Fatos Kongoli, L'ombra dell'altro, Casa editrice Besa, 1999

Fatos Kongoli, L'ombra dell'altro, Casa editrice Besa, 1999

Dino Buzzati, All'idrogeno in Opere scelte, a cura di Giulio Carnazzi, 1998

Fatos Kongoli, Pelle di cane, Casa editrice Argo, 2008

Leal, Luis Magical Realism: Theory, History, Community. Ed.Louis Parkinson Zamora and Wendy B. Faris.Durham, 1995

Giorgio Barberi Squarotti, Landolfi o il potere della letteratura, in "Rapporti", n 22-23, 1981

Y. Panafieu, Dino Buzzati: un autoritratto, Mondadori, Milano 1973

T.S.Eliot, Works of T.S.Eliot, Mobilereference.com, 2010

Dino Buzzati, Le case di Kafka in Opere scelte, a cura di Giulio Carnazzi

Roland Barthes, "Saggi critici", Einaudi, 2002

Roger Caillois, "Nel cuore del fantastico", Rizzoli, 2004

Borghes Jorge L, Ocampo Silvina, Bioy Casares Adolfo, "Antologia della letteratura fantastica", Editori Riuniti, 1980

M. Bontempelli, "Opere scelte", Milano 1978

Fatos Kongoli, "lllusioni nel cassetto", Casa editrice "Toena", Tirana, 2010 